\title{
Los conocimientos detrás del oficio tradicional: conceptos y dimensiones para su análisis
}

\section{The knowledge behind traditional trade: concepts and dimensions for analysis}

\author{
AYALA-RAMIREZ, Suhey†, CASTILLO-GIRON, Víctor Manuel y ANTOINE, Marva Esther Marina \\ Universidad de Guadalajara - Centro Universitario de los Valles. Carretera Guadalajara-Ameca Km. 45.5, 46600 Jal. Méx.
}

ID $1^{\text {er }}$ Autor: Suhey, Ayala-Ramirez / ORC ID: 0000-0003-1079-9605, CVU CONACYT ID: 171341

ID $1^{\mathrm{er}}$ Coautor: Víctor Manuel, Castillo-Giron / ORC ID: 0000-0002-8307-2952, CVU CONACYT ID: 31156

ID $2^{\text {do }}$ Coautor: Marva Esther Marina, Antoine / ORC ID: 0000-0002-8683-6188, CVU CONACYT ID: 369544

DOI: $10.35429 / J O C S .2019 .18 .6 .13 .22$

Recibido: 18 de Enero, 2019; Aceptado 29 de Marzo, 2019

\begin{abstract}
Resumen
Los oficios tradicionales, son modos de producción de bienes materiales. Tienen características particulares que los hacen distintos a otras formas de producción. Su estudio, es relevante toda vez que, encierran una conceptualización de las estructuras socioculturales creadas en el momento histórico en el que éstos surgieron, dadas las relaciones sociales de producción establecidas entre los individuos. Asimismo, dan cuenta de las transformaciones sociales como consecuencia de los cambios vividos por estos modos de producción (Martínez, 2009). Para el caso particular del presente artículo, el estudio se hace desde una perspectiva educativa. Con el objetivo de analizar conceptual los oficios tradicionales, con énfasis en los conocimientos, saberes y modos de transmisión que se movilizados en éstos y del papel de la educación como factor de ruptura, continuidad, transformación y/o consolidación de los mismos. La metodología se deriva del análisis bibliográfico, que permitió hace una aproximación conceptual de los oficios tradicionales, en tanto que es escasa la bibliografía especializada en este tema y con la intención de aportar elementos para análisis empíricos posteriores que permitan comprender el bagaje socioeducativo contenido en estos modos de producción que han sido desvalorizados por el sistema educativo formal.
\end{abstract}

Oficios tradicionales, Educación, Conocimiento tácito artesanal

\begin{abstract}
Traditional trades are a means of producing material goods. They have distinguishing characteristics that differentiate them from other forms of production. Their study is relevant since they conceptualize the sociocultural structures created at a given moment in history, and speak to the social relations forged during production. Similarly, they account for the social transformations that emerge as a consequence of changes experienced by these means of production (Martínez, 2009). The current study is being approached from an educational perspective. Its objective is to carry out a conceptual analysis of traditional trades, with an emphasis on the transfer of explicit and tacit knowledge and the role of education in their rupture, continuity, transformation and/or consolidation. The research methodology is derived from a thematic analysis of scholarly literature that allowed for the conceptual framing of traditional trades. Notwithstanding, there is a limited supply of specialized literature available on this topic, and this study aims to contribute elements for future imperial analysis. Furthermore, the findings will result in a greater understanding of the socio-educational wealth of knowledge contained in these means of production that have been devalued by the formal educational system.
\end{abstract}

Traditional trades, Education, Tacit artisanal knowledge

Citación: AYALA-RAMIREZ, Suhey, CASTILLO-GIRON, Víctor Manuel y ANTOINE, Marva Esther Marina. Los conocimientos detrás del oficio tradicional: conceptos y dimensiones para su análisis. Revista de Sociología Contemporánea. 2019. 6-18: 13-22.

\footnotetext{
$\dagger$ Investigador contribuyendo como primer autor.
} 


\section{Introducción}

Los oficios tradicionales, son modos de producción de los bienes materiales que las personas requieren para su subsistencia. Tienen características particulares que los hacen distintos a otras formas de producción. El estudio y análisis de dichos oficios, es relevante toda vez que, en ellos se encierra toda una conceptualización de las estructuras socioculturales creadas en el momento histórico en el que éstos surgieron, dadas las relaciones sociales de producción establecidas entre los individuos. Asimismo, dan cuenta de las transformaciones sociales como consecuencia de los cambios vividos por estos modos de producción (Martínez, 2009).

En este sentido, los oficios tradicionales pueden ser analizados desde diversas perspectivas disciplinares. Según Martínez (2009), desde la economía y la historia se resalta el análisis de las actividades tradicionales, "las relaciones sociales que se establecen en su organización, su producción, el comercio y la fiscalización desde las esferas de poder gubernamental o institucional". Desde la perspectiva antropológica o etnológica, el interés está en "los descubrimientos o avances tecnológicos que posibilitaron el conocimiento de procedimientos o técnicas que permitieron al hombre aprovechar o transformar los recursos naturales". En tanto que para la geografía el análisis se centra básicamente en el trabajo del ser humano y su relación con el medio físico.

Para el caso particular del presente documento, el análisis de los oficios tradicionales se hace desde una perspectiva educativa. Con el objetivo de analizar conceptualmente los oficios tradicionales, con énfasis en los conocimientos, saberes y modos de trasmisión que se movilizados en éstos y del papel de la educación como factor de ruptura, continuidad, transformación y/o consolidación de los mismos. La metodología se deriva del análisis bibliográfico, que permitió hace una aproximación conceptual de los oficios tradicionales, en tanto que es escasa la bibliografía especializada en este tema y con la intención de aportar elementos para análisis empíricos posteriores que permitan comprender el bagaje socioeducativo contenido en estos modos de producción que han sido desvalorizados por el sistema educativo formal.
Además de esta introducción, el artículo se estructura en cinco apartados y unas conclusiones generales. En el primer apartado se establecen los conceptos básicos sobre el oficio tradicional y sus características entre lo domestico y lo industrial. El segundo apartado da cuenta de las transformaciones de estas actividades económicas, las estratégicas que les han permitido adaptarse y los principales retos a los que se enfrentan. Las formas de trasmisión de los oficios tradicionales y su riqueza pedagógica, se analiza en el tercer apartado, mientras que, en el cuarto, se discute el papel de la educación formal en la fortalecimiento, continuidad o ruptura de estos oficios. Por su parte en el quinto apartado, se establece una diferenciación entre las diversas actividades económicas (de lo doméstico a las profesiones). Considerando aspectos relacionados con el tipo de conocimientos, saberes, habilidades y tiempos de aprendizaje que se manifiestan en cada uno de estos niveles de producción.

Se finaliza con una serie de conclusiones dirigidas a destacar los aspectos relevantes de los oficios tradicionales, su riqueza e importancia para ser considerados, rescatados e incluidos por los sistemas institucionalizados del trabajo y la educación, por ser en muchos de los casos la única oportunidad de formación que han tenido las persona que los ejercen y el valor que tienen para impulsar procesos de innovación.

\section{Los oficios tradicionales}

Un oficio es entendido "como el conjunto de actividades que realiza una persona para transformar un material en un bien o servicio, para lo cual moviliza una serie de habilidades, saberes y conocimientos, utiliza herramientas rudimentarias o con tecnología muy básica. $\mathrm{Su}$ destreza está ligada al tiempo con que se practican dichas actividades y a la experiencia adquirida con el ejercicio. Es construido socialmente y constituye un elemento constructor de identidad" (Ayala, 2016, pp. 19). De esta manera, los oficios tradicionales tienen características distintivas, entre las que destacan: La capacidad de crear "algo" (cosa o bien), quien ejerce el oficio conoce y domina todo el proceso de producción. El producto creado o construido es conocido como "hecho a mano", es decir una sola persona lo elabora de principio a fin $\mathrm{y}$ difícilmente se pueden tener dos productos idénticos (en contraste con la producción industrial). 
Al tener el dominio de todo el proceso de producción, quien ejerce el oficio tiene la capacidad de incidir en la modificación, adecuación o cambio en las herramientas o procesos para mejorar la producción.

La forma de trasmisión y aprendizaje del oficio, permite que creatividad de quien posee el oficio se desarrolle y a su vez esto le da la oportunidad de desarrollar modificaciones $\mathrm{o}$ adaptaciones en los modelos de sus productos en función de la demanda o gustos de los consumidores. La transmisión de generación en generación del oficio, permite crear redes de aprendizaje y colaboración.

Por su parte, lo tradicional de los oficios, no refiere a lo atemporal del propio concepto (Martínez, 2009), sino que, en este caso en particular, al ser los oficios una actividad humana creadora de estructuras sociales, que a su vez es construida por el propio contexto. Los oficios son tradicionales en tanto que, no se quedaron intactos en el tiempo, sino que van evolucionando como producto de la actividad humano, sin embargo, son herederos de conocimientos, saberes, habilidades, técnicas y experiencias más o menos antiguas. Y los avances que se pueden visualizar en los oficios no son necesariamente "progresos tecnológicos destacables, sino mejoras o adaptaciones de los procedimientos o las herramientas" (Martínez, 2009). En este sentido, dice Martínez (2009) que lo tradicional no es lo anclado en un momento puntual del pasado, sino lo capaz de permanecer al adaptarse. Por lo tanto, los oficios tradicionales son aquellos modos de producción que se originaron en un contexto histórico, social, cultural y económico especifico, pero que tuvieron la capacidad de adaptarse/transformarse a los cambios, logrando subsistir al paso del tiempo y conservandose en esencia.

\section{Los oficios tradicionales: entre lo domestico y la industria}

Aun cuando más adelante se presentará un análisis sobre las diferencias entre las diversas actividades económicas.

\footnotetext{
${ }^{1}$ Con la explicacion de este ejemplo no se pretender simplificar todo el proceso, tiempo y factores sociales, economcios y culturales que se ponen en juego para que la actividad se transforme, solo se intenta ejemplicar cómo una actividad puede transitar entre diversas formas de producción.
}

Es importante ver como muchos de los oficios tradicionales se originan en el ámbito doméstico, luego derivado de diversos factores se van transformando y se convierten en oficios, algunos de los cuales se consolidan como tales y se quedan en ese nivel. Algunos otros se ven influenciados por diversos factores que los llevan a la industrialización.

En este último caso, puede suceder que la actividad al industrializarse desaparezca como oficio o que se conserve como tal. Es decir que la producción de ciertos materiales o productos puede darse en diversas formas (como oficio tradicional y como producto industrial).

Ahora bien, pasar por estas tres formas de producción no es de manera inmediata ni automática, es un proceso donde confluyen diversos factores: el producto que se desarrolla (si es de primera necesidad o no) y su demanda (prioridad de los consumidores), cuestiones de género, la forma de trasmisión de los conocimientos y saberes de dicha actividad, la salida del espacio privado al espacio público, el desarrollo tecnológico, los cambios en el mercado, los costos para el consumidor y para el productor, entre otros.

Un ejemplo de lo anterior, es la producción de pan, esta actividad era tradicionalmente femenina y doméstica, sin embargo, por la relevancia y demanda del producto (el pan se consume como parte de la dieta básica de la humanidad), en la medida que sale del espacio domestico al espacio público (los talleres se fundan fuera de las actividades propias del hogar), es retomado por los hombres, con ello se transforma a un oficio masculino. Con los avances tecnológicos y los cambios socioeconómicos esta actividad pasa a una producción industrializada. No obstante, se conserva también su producción artesanal1 (Dobles, 1999).

\section{La transformación del oficio tradicional}

Los oficios tradicionales con el paso del tiempo se van enfrentando a diversas circunstancias que ponen en riesgo su permanencia. 
Algunas de éstas son: las condiciones socioeconómicas, la industrialización, situaciones meramente contingentes (el agotamiento o reemplazo de algún recurso natural, el impacto de las innovaciones tecnológicas, crisis económicas, ciertos procesos regionales de reconversión productiva) y la cultura (Ayala, 2016).

Sin embargo, encontramos algunas condiciones propicias que aprovechan para mantenerse vigentes. En decir, los oficios se van adaptando $\mathrm{y}$ transformando a las nuevas condiciones productivas. En ese sentido, se identifican por lo menos cuatro condiciones y/o estrategias de las que se apropian los oficios. La primera son los activos que poseen quien ejerce el oficio y que le permite establecer estrategias para adaptarse a las nuevas condiciones, como son los saberes que se movilizan y el conocimiento y dominio de todo el proceso de producción, con ello son capaces de diseñar nuevos modelos de sus productos. Con lo que son capaces de adaptarse a las demandas de los consumidores y a las novedades o modas. Además, el tener tecnología básica les permite una mayor flexibilidad en su producción.

La segunda estrategia utilizada, es usar transitoriamente la industria ya sea para terminar de aprender el oficio (sobre todo los hijos o aprendices de los maestros del oficio), o conocer técnica o procesos novedosos, aprender a utilizar alguna maquinaria o materia primar, para después adquirir sus propias maquinas y/o instalar su propio taller en sus casas o mejorar el que ya tiene su familia (Dobles, 1999).

La tercera tiene que ver con la materia prima, que en ocasiones es cambiante, en este caso, el efecto negativo se puede minimizar, dado que, al ser poca su producción o sobre demanda, no adquieren grandes cantidades de los materiales que requieren, esto les permite cambiar sin problema (Dobles, 1999). La cuarta condición que les permite adaptarse con cierta facilidad, es el reconocimiento de parte de un sector de consumo que da preferencia y tiene gusto por los productos hechos de manera artesanal.
Bajo este contexto, a pesar de que los talleres de oficios se enfrentan a una fuerte competencia con otros talleres y con las industrias, aquellos que han sido creativos y originales, que han incorporado mejoras en la organización del trabajo y han conservado técnicas y saberes tradicionales, son los que han permanecido vigentes. Es decir que depende en gran medida de la capacidad que tengan los talleres de oficios para adaptarse a las nuevas circunstancias lo que les permite permanecer en el mercado. Considerando que el tiempo y las condiciones de adaptación/transformación de los oficios tradiciones es distinta entre un oficio y otro, ello va a depender de diversos factores que se ponen en juego. La capacidad de adaptarse al cambio, el tipo de producto, las condiciones de las demandas, sin duda son los factores de mayor relevancia.

\section{Trasmisión de los oficios tradicionales}

El análisis de la trasmisión de los oficios tradicionales es relevante, toda vez que nos permite comprender el bagaje y potencial educativo que guardan. En ese sentido, hablar de transmitir el oficio como bien dice Agüero y Cordero (1999) no es mantener las practicas, formas y condiciones idénticas de una persona a otra. Es decir, el oficio es trasmitido de una generación a otra y en ese proceso se van dando cambios por las aportaciones tanto personales de quien recibe el oficio como del propio contexto en que se desarrolla.

Al ser el oficio trasmitido de generación en generación, el primer aspecto de importancia en este proceso, es el papel de la familia. Puesto que es ésta quien dirige la actividad productiva, quien posee el oficio y quien además consume el producto. En esta unidad podemos observar una división del trabajo por género y edad. Los miembros que la conforman puedes incluso participar en otras unidades productivas, no obstante, son parte del oficio familiar. En ese mismo sentido, el oficio es una posesión de la familia, y sus miembros lo llevarán consigo sin importar las condiciones, es decir, si las oportunidades o necesidades los obligan a cambiar el lugar de residencia, el oficio irá con ellos y lo trasmitieran a sus hijos (Agüero y Cordero, 1999). 
El segundo aspecto a considerar en la trasmisión del oficio, tiene que ver con las fuentes de aprendizaje, en ese sentido y según lo planteado por Landini (2010) y Nuñez (2004) podemos identificar por lo menos tres.

1. Aprendizajes producidos en la niñez: al ser el hogar un espacio de producción y reproducción del oficio, los niños son parte del propio taller, es decir, están inmersos en las actividades propias del proceso de producción e incluso de la administración y comercialización del producto. El niño juega e imita las actividades del taller. Por ello, con el tiempo va adquiriendo saberes y destrezas que incluso ni él mismo pueden explicar cómo los adquirió, pero le permiten ejercer el oficio.

2. Cambios externos: cuando surgen cambios, modificaciones o aparecen nuevos actores externos al propio oficio pero que tienen influencia en el mismo, se tienen que hacer adecuaciones, transformaciones, adaptaciones o incluso adopciones. Esto puede ser derivado de nuevas prácticas o dinámicas del mercado y/o productos, o modificaciones en las materias primas, con lo que se tienen que hacer pruebas y se experimenta, con ello se generan nuevos aprendizajes y con el paso del tiempo, nuevos conocimientos.

3. Saberes locales: en este caso se refiere a los saberes que se transmiten a lo largo de décadas de padres a hijos, dentro de una comunidad. Mismo que pueden o no estar relacionados directamente con el oficio, sin embargo, que permiten complementar el ejercicio del mismo. Estos saberes son considerados tradiciones y en muchos de los casos se van perdiendo con el paso del tiempo, dado que su trasmisión es de manera oral y no se dejan evidencias escritas de los mismos.

El tercer aspecto que se debe analizar en la trasmisión del oficio son los modos en que se trasmiten los conocimientos y saberes. En este sentido, encontramos que una característica distintiva en estos modos de producción es que la trasmisión se hace de generación en generación, en entornos informales y vinculados con situaciones reales y prácticas.
Se utilizan básicamente cuatro medios en su trasmisión (Ayala, 2016; Gómez y Gómez, 2006; Landini, 2010 y Luévano, 2008):

1. La oralidad: los conocimientos se trasmiten de manera verbal, se explicando de manera detallada cada paso del proceso de producción. El conocimiento no está explicitado de manera escrita. No se cuenta con manuales de procedimiento.

2. La observación: el aprendiz debe observar cuidadosamente cómo hace el otro cada actividad del proceso de producción. Lo que el maestro no explica con palabras lo muestra con la práctica y el aprendiz debe poner mucha atención para captar todos los gestos, movimientos, tiempos y formas.

3. La imitación: después de escuchar las instrucciones y de observar al maestro realizar todas y cada una de las actividades que conlleva la producción, el aprendiz debe imitar los movimientos y gestos.

4. La práctica: el aprendiz debe practicar una y otra vez hasta dominar todas las actividades que se deben realizar en el proceso de producción. En la práctica se centra el proceso de aprendizaje del oficio.

Estos cuatro medios de trasmisión que son fundamentales para la enseñanzaaprendizaje de un oficio, no se dan de manera separada ni en un orden específico, sino más bien, se combinan unos con otros, en momentos y orden diversos. Dependerá de la dificultad de cada actividad, de las habilidades del aprendiz y del maestro y del tiempo que se lleve aprender a dominar cada fase del proceso de producción, el medio o combinación de medios que son utilizados para la trasmisión de los conocimientos.

\section{Conocimientos y saberes de los oficios tradicionales}

En los oficios tradiciones existe un cumulo de saberes, prácticas, técnicas, conocimientos y/o cosmovisiones que responden a problemas específicos de las actividades de cada oficio y también al contexto sociocultural de los mismos. 
De manera particular, en los oficios tradicionales se generan conocimientos forjados en la vida cotidiana de quien los ejerce, son producto de las interacciones con los otros y en la práctica constante del ejercicio. Para Ayala (2016) estos conocimientos son llamados conocimientos tácitos artesanales, puesto que “... la destreza de quien los ejerce está ligada a la experiencia adquirida con el ejercicio vinculado a la práctica cotidiana. Está relacionado al "saber hacer" y está vinculado al contexto y a la construcción de subjetividades que abarcan creencias, mitos, intuiciones, modelos mentales y habilidades técnicas. Por otra parte, los conocimientos de los oficios son artesanales (artes de hacer) puesto que se generan en la práctica cotidiana de quien los ejerce, y refieren a actividades y procedimientos populares. Además, al ser construcciones sociales representan la suma de la racionalidad creativa del colectivo, y en este sentido son comunicados a través de su propio lenguaje, símbolos, movimientos, tiempos y acciones." (Ayala, 2016, pp. 25).

En ese sentido, estos conocimientos son enriquecidos y reconfigurados a partir de la incorporación de avances tecnológicos y conocimientos explícitos. Para Landini (2010), surgen de la experiencia de una comunidad y tienen una carga fuerte de interpretaciones y creencias personales, se caracterizan por tener un énfasis práctico y vínculo con el trabajo manual y físico.

En el caso del Saber hacer, que está ligado a la generación y trasmisión de conocimientos tácitos artesanales en los oficios. Es necesario enfatizar en las implicaciones de este concepto, es decir, cualquier persona puedes saber hacer una "cosa", no obstante en el caso de quien ejerce el oficio, no solo sabe hacer tal o cual "cosa" o producto, sino que además de eso, es capaz de tener un conocimiento completo que le permite construir esa "cosa", sabe las reglas, los principios, máximas y recetas, sabe manipular los instrumentos, herramientas, utensilios (Vega, 2001).

En ese sentido, según Vega (2001), se debería de precisar el concepto, y en lugar de llamarlo Saber hacer, sería más preciso decir Saber cómo hacer.
Con esto daríamos cuenta de que quien ejerce el oficio, es capaz de crear algo, porque tiene tanto un conocimiento de cómo se hace (medios) y la habilidad para hacerlo. Entonces, en los oficios tradicionales encontramos personas que saben cómo hacer un producto y también son capaces de hacerlo, y además pueden enseñar a otros cómo hacerlo, para ello debe ser capaz de describir correctamente el proceso, paso a paso.

En ese sentido, en los oficios tradicionales estaríamos hablando no de un aprender haciendo, sino más precisamente de hacer aprendiendo (Dewer citado por Sontoni, 1994). Dado que este aprendizaje se da de manera un tanto involuntaria, es decir, el objetivo o intención no es precisamente el aprendizaje, sino el hacer tal o cual cosa. En ese sentido, el aprendiz no pueda dar cuenta de en qué momento aprendió a realizar cada parte del proceso, cuando menos lo piensa ya es capaz de dominar el proceso de producción y no repara en reflexionar sobre cómo logró aprender.

Con base a todo lo anterior podemos decir que, en los oficios tradicionales, existen dos dimensiones en la formación y trasmisión del oficio. La primera es la técnica, se centra en la construcción del propio objeto, incluyendo el dominio de las reglas y el conocimiento experto. La segunda se realiza a través de instrucciones precisas que marcan los parámetros, procedimientos, tiempos, etcétera, para ejecutar las operaciones necesarias en el proceso de elaboración de algún producto.

\section{La educación formal como factor de ruptura/continuidad del oficio}

La formación de quien ejerce el oficio debe entenderse como parte de los procesos de educación y como parte de un contexto del desarrollo de políticas educativas. $\mathrm{Si}$ consideramos la educación como la define Gómez y Gómez (2006):

"La educación, se concibe como un proceso de intervención con intencionalidad que pretende el perfeccionamiento del sujeto a través de un proceso gradual, activo integral $y$ permanente (Yzunza, 2000). Coincidiendo con Edgar Morín (1999), cuando asevera que "la condición humana, debería ser objeto esencial de cualquier educación". 
También se considera como finalidad de la educación a la perpetuación de una tradición establecida y la posibilidad de un futuro diferente (Ardoino, 1980)."

Bajo este contexto, la educación se da en distintos entornos, de diversas maneras y con diferentes propósitos. Con el tiempo se ha convertido en una responsabilidad del Estado, y en ese sentido se establecen políticas educativas donde priman ideas de progreso, libertad, igualdad y desarrollo las cuales fueron llevadas a la práctica en mediación principal por las instituciones educativas, donde se da prioridad al saber científico sobre los saberes tradicionales (Nuñez, 2004). Sin embargo, en la práctica, la educación no solo se ha desarrollado en el ámbito formal y no formal, también se ha desarrollado en entornos informales a los que una parte importante de la población han accedido como única opción o como parte de la tradición familiar y local. No obstante, para el sistema de educación formal y para las instituciones del trabajo, la formación que se da como parte de este tipo de educación no es considerada y más bien es desvalorizada.

Como se ha mencionado en apartados anteriores, los oficios tradicionales contienen una carga importante de conocimientos, saberes, habilidades y destrezas, con lo que es considerada educación informal. Quien ejerce un oficio ha tenido acceso a esa educación, en algunos casos solo a ésta y en otros también a la educación formal básica o superior y/o a la educación no formal. Por ello, es relevante conocer los tipos de educación a la que han tenido acceso las personas que ejercen el oficio y cómo ésta ha influido como ruptura, continuidad y/o transformación del oficio.

Generalmente quien ejerce el oficio tiene un desenvolvimiento educativo basado en el carácter elemental de la educación primaria (Agüero y Cordero, 1999; Ayala, 2016 y Dobles, 1999). Lo que los lleva a saber leer, escribir y hacer operaciones matemáticas básicas (lo que se conocía como las tres " $r$ ", leer, escribir y hacer cuentas). Ello tiene poca incidencia en la ruptura de los oficios, más bien, permiten fortalecer áreas de oportunidad, y tampoco les permite desarrollar otras ocupaciones o actividades alternativas o distintas al oficio de la familia, lo que le da continuidad al mismo.
No obstante, el acceso a la educación media superior y superior, dota a las personas con competencias para desarrollar actividades técnicas y profesionales. En este caso, se tiene poca evidencia documentada que permita conocer que sucede con los oficios. Se tiene el estudio de Ayala (2016), donde se afirma que el oficio de tequilero se fortalece y continua cuando los maestros tequileros tienen acceso a la educación superior. Sin embargo, no continua en las mismas condiciones, sino que se fortalece y sufre transformaciones y tampoco todos los miembros de la familia aprenden el oficio.

En ese sentido, es relevante tener mayores referentes empíricos, para conocer qué pasa con otros oficios y cómo convergen la educación formal y la informal, analizando tanto lo derivado de lo impartido en las aulas como también de lo que se inculca en los talleres / hogares. Resaltando el papel de la trasmisión intergeneracional de los oficios (Agüero $\mathrm{y}$ Cordero, 1999).

Es evidente la inexistencia de políticas o estrategias que permitan proteger, promover y conservar los conocimientos tácitos artesanales que son transferidos de generación en generación y que contienen un gran valor tanto para el desarrollo económico, como cultural e incluso como insumo de la innovación (Ayala, 2016). Por ellos, se reconoce la importancia de aprovechar la riqueza contenida en éstos conocimientos que aun cuando no pueden ser medidos, validados, objetivados y generalizados como lo exige la ciencia positivista (Nuñez, 2004), tienen relevancia en diversos ámbitos como el social, cultual, familiar y económico.

\section{Niveles de educación: del oficio a la profesión}

A menudo el termino oficio es utilizado como sinónimo de otros modos de producción y actividades económicas tradicionales o no. Es común encontrarse tanto en la bibliografía especializada como en el lenguaje cotidiano con expresiones donde no se hace diferencia entre ocupaciones, actividades domésticas, oficios, actividades técnicas y profesiones. Es verdad que los límites entre estas pueden ser confusos y tal vez inexistente, sin embargo, es posible identificar algunas características que permitan hacer una diferenciación entre ellas. 
De esta manera, se han identificado algunos criterios que pueden ayudar a identificar sus particularidades, considerando como centro aspectos educativos como son: los conocimientos y saberes que se movilizan en cada una de las actividades económicas, los modos de trasmisión, el tipo de educación, el tiempo de formación.

Así como algunos aspectos relacionados con la producción como son: el tipo, la capacidad, la infraestructura utilizada en la producción.

Como se puede observar en la Tabla 1, los tipos de conocimientos que son movilizados en las actividades económicas, son los mismo, sin embargo, lo que cambia es cantidad y prioridad que se le da a cada tipo, es decir, mientras que en el oficio se da prioridad y se utiliza en mayor medida los conocimientos tácitos, pero también, aunque en menor cantidad, se movilizan conocimientos explícitos.

En el caso de la profesión, se da prioridad los conocimientos explícitos y aunque si son utilizados los tácitos, para los profesionistas éstos no son importantes en su formación.

En cuanto a la escolaridad, podemos observar que las ocupaciones domésticas son las menor o nula escolaridad y tecnificación, esto va aumentando para cada actividad hasta llegar a la alta escolaridad y tecnificación de las profesiones.

Esto se ve relacionado con el tipo y capacidad de producción, donde es menor en la ocupación doméstica y de igual manera va aumentando.

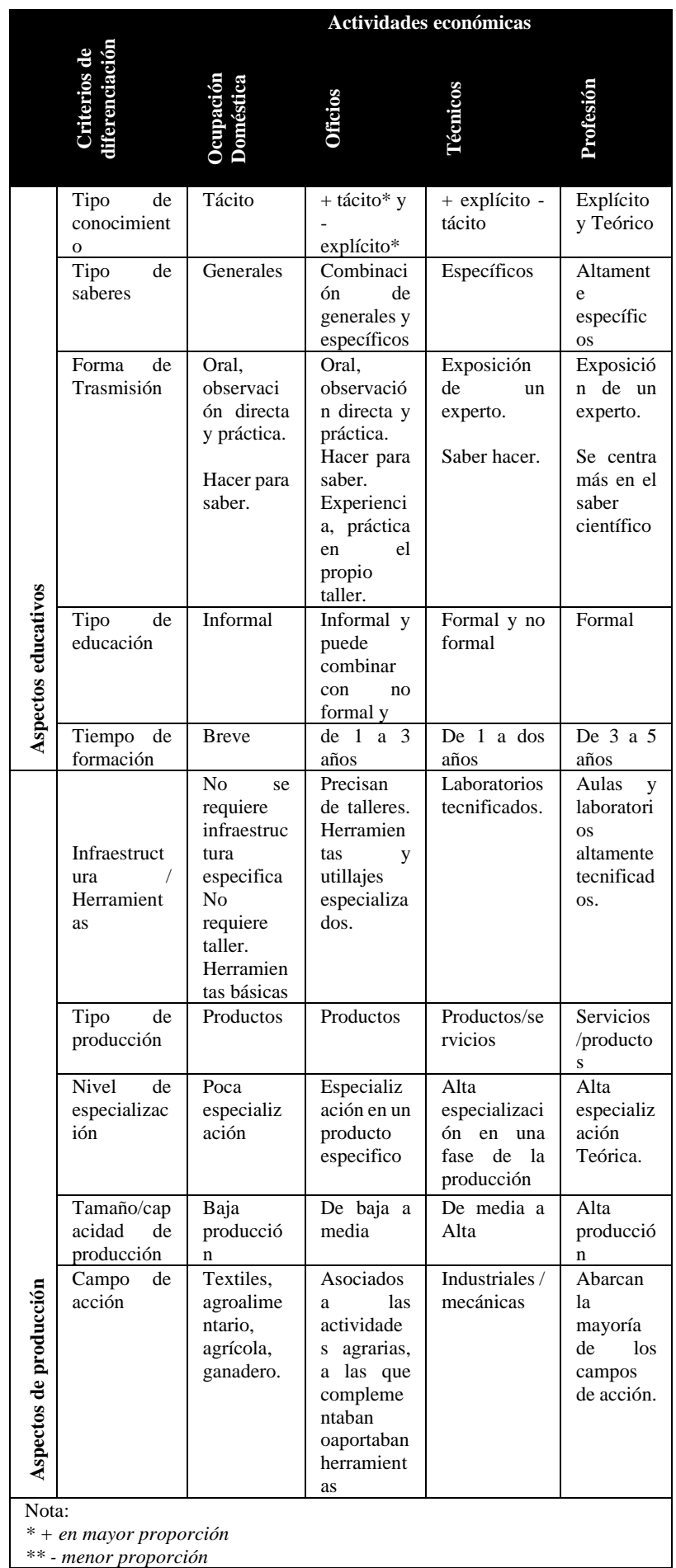

Tabla 1 Características de las actividades económicas Fuente: elaboración propia

Otra de las diferencias que se destaca, es el nivel de institucionalización. Es decir, entre más profesionalizante la actividad mayor es la institucionalización tanto de la educación, como de los procesos de producción. En este aspecto, se da prioridad al conocimiento científico y positivista y se desvalorizan los conocimientos tácitos, la experiencia personal, el bagaje de la vida cotidiana. 
En este sentido, al visualiza estas diferencias significativas entre las diversas actividades económicas o modos de producción, abren el panorama para el estudio de las particularidades de las mismas con diversas finalidades, en el caso del presente artículo, el interés es determinar que en términos educativos las diferencias son importantes para analizar las formas como las personas se van apropiando de conocimientos y saberes que son generados, trasmitidos y conservados a través del tiempo como medios de preservación y subsistencia.

\section{Conclusiones}

Los oficios tradicionales analizados desde una perspectiva educativa, son formas de producción y reproducción de una pedagogía que se basa en el razonamiento y establecimiento de los propósitos de una actividad, que a su vez es verificada y rectificada continuamente. Que, además, también es evaluada mediante un producto final que debe cumplir con la exigencia del consumidor, con ello quien ejerce el oficio debe demostrar con este producto que domina todo el proceso, cuida las salidas terminales y en general esta "con un ojo al taller y otro a la calle" Santoni (1994).

Los modos de trasmisión de los oficios tradicionales, son una forma de educación que permite a las personas no solamente realizar un trabajo o una actividad específica, sino que también les permite desarrollar su creatividad, la habilidad inventiva, crear nuevas formas y herramientas. Tal como lo asegura Santoni (1994) de los maestros del oficio surgieron grandes inventos.

Con base a lo anterior, y en términos generales, lo que encontramos en los oficios tradicionales es a lo que se aspira en las instituciones de educación; que los individuos cultiven valores y sean capaces de innovar y ofrecer alternativas de convivencias armónicas entre los seres humanos y de éstos con la naturaleza. En ese sentido, encontramos que la educación formal (institucionalizada) juega un papel importante en la consolidación, fortalecimiento y continuidad del oficio, puesto que permite consolidar habilidades y competencias de quien ejerce el oficio y le permite hacer adaptaciones significativas en el proceso de producción.
No obstante, el valor intangible de los oficios tradicionales, (educativo, pedagógico, cultural, etcétera). Es importante considerar que éstos se enfrentan a problemas severos económicos, de competitividad con las grandes industrias y de disminución en la demanda de sus productos. Aquí es donde se deben fortalecer los procesos de creatividad e innovación, en este caso no necesariamente en el propio producto, pero si en la formación de los consumidores que buscan productos con riqueza cultural y reconocen el valor de lo hecho a mano.

\section{Referencias}

Agüero, J., \& Cordero, R. (1999). La Transmisión Intergeneracional de los Oficios de Cañero y de Productor de Granos Básicos en el Mundo Rural Costarricense (18501960). Anuario de Estudios Centroamericanos, 25(1), 111-138. Retrieved from URL:

https://www.jstor.org/stable/25661314

Ayala, S. (2016). Destilando Saberes. Los gajes del oficio tequilero. Universidad de Guadalajara. México.

Dobles Trejos Cecilia. (1999). Hilvanando Historias una Aproximación al Conocimiento del Oficio de Lacostura 1900-1960. Anuario de Estudios Centroamericanos, 25, 61-81. Retrieved

from https://www.jstor.org/stable/25661312?se $\mathrm{q}=1$ \#metadata_info_tab_contents

Gómez-Espinoza, J. A. G., Gerardo. (2006). Saberes tradicionales agrícolas indígenas y campesinos: rescate, sistematización e incorporación a la IEAS. Ra Ximhai, 2, 97-126. Retrieved

from http://www.redalyc.org/articulo.oa?id=46 120106

Landini, F. (2010). La dinámica de los saberes locales y el proceso de localización del saber científico. Aportes desde un estudio de caso. Cuadernos de Desarrollo Rural, 7(65), 1940.

Retrieved from http://www.redalyc.org/articulo.oa?id=11 716958001

Luévano Bustamante Guillermo. (2008). Con las manos en la masa Los sindicatos de panaderos de San Luis Potosí, 1915-1934. Desacatos, 27, 169-184. 
Martínez, Javier (2009). La revalorización y promoción de los oficios tradicionales. CUADERNOS, 22. Retrieved from http://www.xiloca.org/data/Bases\%20dato s/Cuadernos/C_22_05_18.pdf

Núñez R. Jesús (2004). Saberes Y Educación. Una mirada desde las culturas rurales. Revista Digital ERural, Educación, Cultura y Desarrollo Rural,2. Retrieved from redler.org/saberes_educacion_culturas_rurales.pdf

Santoni, Antonio. (1994). Nostalgia Del Maestro Artesano. Universidad Autónoma de México. México.

Vega Encabo Jesús. (2001). Reglas, Medios, Habilidades. Debates en Torno al Análisis de "5 Sabe Cómo Hacer X.". Crítica, Revista Hispanoamericana de Fil, 33, 3-40. Retrieved from https://www.jstor.org/stable/40104881?se $\mathrm{q}=1$ \#metadata_info_tab_contents 